PROCEEDINGS OF THE

AMERICAN MATHEMATICAL SOCIETY

Volume 128, Number 1, Pages 49-51

$\mathrm{S}$ 0002-9939(99)05138-2

Article electronically published on May 27, 1999

\title{
SETS OF $p$-POWERS AS CONJUGACY CLASS SIZES
}

\author{
JOHN COSSEY AND TREVOR HAWKES
}

(Communicated by Ronald M. Solomon)

\begin{abstract}
We show that any finite set of powers of a fixed prime $p$ which includes 1 can be the set of conjugacy class sizes of a $p$-group of nilpotency class

2. This corresponds to a result of Isaacs for degrees of irreducible characters.
\end{abstract}

\section{INTRODUCTION}

If $G$ is a finite group, we denote by $c d(G)$ and $c s(G)$ the sets of numbers which occur as the degrees of the irreducible characters of $G$ and as the sizes of the conjugacy classes of $G$ respectively. Results about the set of irreducible character degrees sometimes correspond to similar results about the set of conjugacy class sizes. Here we give an instance of such a correspondence. Let $p$ be a prime, and let $\mathcal{S}$ be a set of powers of $p$ containing $p^{0}=1$. Isaacs proves in [1] that there is a $p$-group $P$ of class 2 for which $c d(P)=\mathcal{S}$. We will prove an analogous result for the set of conjugacy class sizes.

Theorem. Let $p$ be a prime and $\mathcal{S}$ a finite set of p-powers containing 1 . Then there exists a p-group $P$ of class 2 with the property that $\operatorname{cs}(P)=\mathcal{S}$.

\section{Proof of The Theorem}

The set of $G$-conjugates of an element $x$ of a group $G$ will be denoted by $x^{G}$ and the minimal number of generators of $G$ by $d(G)$.

Denote the given set $\mathcal{S}$ of powers of the prime $p$ by

$$
\mathcal{S}=\left\{p^{\alpha_{0}}, p^{\alpha_{1}}, \ldots, p^{\alpha_{n}}\right\}
$$

with the convention that $0=\alpha_{0}<\alpha_{1}<\alpha_{2}<\ldots<\alpha_{n}$. We will construct a group $P_{\mathcal{S}}$ satisfying the following conditions:

- $c s\left(P_{\mathcal{S}}\right)=\mathcal{S}$,

- $P_{\mathcal{S}}$ has class 2 , exponent $p$ if $p$ is odd, and exponent 4 if $p=2$, and

- $d\left(P_{\mathcal{S}}\right)=\alpha_{n}+1$.

The construction requires some ideas from the theory of varieties of groups. We refer the reader to Hanna Neumann's book [2] for the meaning of our notation and for any unexplained ideas and results.

Received by the editors March 24, 1998.

1991 Mathematics Subject Classification. Primary 20D60.

The authors wish to acknowledge the support of the Australian Research Council and the Engineering and Physical Sciences Research Council. They are also grateful to the referee for pointing out a gap in the proof and an elegant way to fill the gap.

(C)1999 American Mathematical Society 
When $p$ is odd, $\mathfrak{V}_{p}$ will denote the variety of $p$-groups of class at most 2 and exponent $p$ (note that since $p$ is odd, $\mathfrak{V}_{p}$ contains nonabelian groups); $\mathfrak{V}_{2}$ will denote the variety generated by the dihedral group of order 8 . For a positive integer $n$, we denote by $F_{n}$ the free group of rank $n$ in the variety $\mathfrak{V}_{p}$ and by $A_{n}$ the elementary abelian $p$-group of rank $n$. Note that $F_{n} / \Phi\left(F_{n}\right)$ is isomorphic to $A_{n}$, where $\Phi\left(F_{n}\right)$ is the Frattini subgroup of $F_{n}$. Note also that $F_{n}^{\prime} \leq \zeta\left(F_{n}\right)$ (since $F_{n}$ has class 2) and that $p$ th powers are central (since they are trivial if $p$ is odd and squares are central in the dihedral group of order 8). Thus $\Phi\left(F_{n}\right) \leq \zeta\left(F_{n}\right)$. We will need the following crucial fact about $F_{n}(n \geq 2)$ :

Elements $x$ and $y$ of $F_{n}$ that are independent modulo $\Phi\left(F_{n}\right)$ do not commute. To see this, observe that $\langle x, y\rangle$ is a free group of rank 2 in $\mathfrak{V}_{p}$ with $x$ and $y$ as free generators by [2], Theorem 42.31. If $x$ commuted with $y$, then $[x, y]=1$ would be a law in $\mathfrak{V}_{p}$ by [2], Corollary 13.25, and then $\mathfrak{V}_{p}$ would be abelian, a contradiction. It follows, in particular, that $\zeta\left(F_{n}\right)=\Phi\left(F_{n}\right)$.

We will also need the following special case of the varietal product (see [2], Section 1.8, for a detailed account of this construction). Suppose that $X, Y$ are groups in $\mathfrak{V}_{p}$. Then the $\mathfrak{V}_{p}$-product $X * \mathfrak{V}_{p} Y$ of $X$ and $Y$ is defined by

$$
X * \mathfrak{V}_{p} Y=(X * Y) / V,
$$

where $X * Y$ is the free product of $X$ and $Y$ and $V$ is the verbal subgroup of $X * Y$ corresponding to $\mathfrak{V}_{p}$. We note that $V \leq[X, Y]$ since $[X, Y]$ is the kernel of the natural homomorphism of $X * Y$ onto $X \times Y$, and so by definition

$$
[X, Y] \leq \zeta\left(X * \mathfrak{v}_{p} Y\right)
$$

Next, let $x \in X \backslash \Phi(X)$ and $y \in Y \backslash \Phi(Y)$, and let $M, N$ be maximal subgroups of $X$ and $Y$ respectively with $x \notin M, y \notin N$. By [2], Theorem 18.42, there is an epimorphism of $X * \mathfrak{V}_{p} Y$ onto $(X / M) * \mathfrak{V}_{p}(Y / N)$. Since $X / M$ and $Y / N$ have order $p$, the product $(X / M) * \mathfrak{V}_{p}(Y / N)$ is $\mathfrak{V}_{p}$-free of rank 2 if $p$ is odd (by [2], Corollary 18.43), while if $p=2$, it is easy to see that $(X / M) * \mathfrak{V}_{p}(Y / N)$ is the dihedral group of order 8. In either case, the images of $x$ and $y$ in $(X / M) *_{\mathfrak{V}_{p}}(Y / N)$ do not commute, whence $[x, y] \neq 1$ in $X *_{\mathfrak{V}_{p}} Y$. We will need the following consequences of this fact. Let $X$ and $Y$ be nontrivial groups in $\mathfrak{V}_{p}$ and set $G=X *_{\mathfrak{V}_{p}} Y$; then $\zeta(G)=\Phi(X) \Phi(Y)[X, Y]=\Phi(G)$ and so $d(G / \zeta(G))=d(X)+d(Y)$.

We will now show by induction on $|\mathcal{S}|=n+1$ that, for $\mathcal{S}=\left\{p^{\alpha_{0}}, p^{\alpha_{1}}, \ldots, p^{\alpha_{n}}\right\}$ with $0=\alpha_{0}<\alpha_{1}<\alpha_{2}<\ldots<\alpha_{n}$, we can construct a group $P_{\mathcal{S}}$ in $\mathfrak{V}_{p}$ on $\alpha_{n}+1$ generators satisfying $c s\left(P_{\mathcal{S}}\right)=\mathcal{S}$ and also, when $n>0, \zeta\left(P_{\mathcal{S}}\right)=\Phi\left(P_{\mathcal{S}}\right)$. If $n=0$, we choose $P_{\mathcal{S}}=A_{1}$, for which we clearly have $c s\left(P_{\mathcal{S}}\right)=\mathcal{S}$ and $d\left(P_{\mathcal{S}}\right)=1=\alpha_{0}+1$. For $n=1$ and $\mathcal{S}=\left\{1, p^{\alpha}\right\}$, we choose $P_{\mathcal{S}}=F_{\alpha+1}$. In this case, we have $d\left(P_{\mathcal{S}}\right)=$ $\alpha+1$ and $\zeta\left(P_{\mathcal{S}}\right)=\Phi\left(P_{\mathcal{S}}\right)$ since $P_{\mathcal{S}}$ is free in $\mathfrak{V}_{p}$. To see that $c s\left(P_{S}\right)=S$, suppose that $x$ is chosen in $P_{\mathcal{S}}$ but not in $\Phi\left(P_{\mathcal{S}}\right)$. From earlier observations, we have $C_{P_{\mathcal{S}}}(x)$ $=\Phi\left(P_{\mathcal{S}}\right)\langle x\rangle$ and therefore $\left|P_{\mathcal{S}} / C_{P_{\mathcal{S}}}(x)\right|=p^{\alpha}$. It follows immediately that $P_{\mathcal{S}}$ has only the two conjugacy class sizes 1 and $p^{\alpha}$, as required.

Now let $\mathcal{S}=\left\{1, p^{\alpha_{1}}, \ldots, p^{\alpha_{n}}\right\}$ with $n \geq 2$, and set $\mathcal{S}^{*}=\left\{1, p^{\alpha_{2}-\alpha_{1}}, \ldots, p^{\alpha_{n-1}-\alpha_{1}}\right\}$. Since $\left|\mathcal{S}^{*}\right|=n-1$, our inductive hypothesis yields a group $P_{\mathcal{S}^{*}}$ in $\mathfrak{V}_{p}$ on $\alpha_{n-1}-\alpha_{1}+1$ generators with $\operatorname{cs}\left(P_{\mathcal{S}^{*}}\right)=\mathcal{S}^{*}$ and $\zeta\left(P_{\mathcal{S}^{*}}\right)=\Phi\left(P_{\mathcal{S}^{*}}\right)$. We now set

$$
P_{\mathcal{S}}=F_{\alpha_{1}} * \mathfrak{V}_{p}\left(P_{\mathcal{S}} \times A_{\left(\alpha_{n}-\alpha_{n-1}\right)}\right) \text {, }
$$

the free $\mathfrak{V}_{p}$-product of $F_{\alpha_{1}}$ and $P_{\mathcal{S}^{*}} \times A_{\left(\alpha_{n}-\alpha_{n-1}\right)}$. Observe that $\zeta\left(P_{\mathcal{S}}\right)=\Phi\left(P_{\mathcal{S}}\right)$ and that $d\left(P_{\mathcal{S}}\right)=d\left(F_{\alpha_{1}}\right)+d\left(P_{\mathcal{S}^{*}} \times\left(A_{\left(\alpha_{n}-\alpha_{n-1}\right)}\right)\right)$ by our remark above. In particular, 
$d\left(P_{\mathcal{S}}\right)=\alpha_{1}+\alpha_{n-1}-\alpha_{1}+1+\alpha_{n}-\alpha_{n-1}=\alpha_{n}+1$. To complete the induction, it remains to show that $c s\left(P_{\mathcal{S}}\right)=\mathcal{S}$.

For notational convenience, set $X=F_{\alpha_{1}}$ and $Y=P_{\mathcal{S}^{*}} \times A_{\left(\alpha_{n}-\alpha_{n-1}\right)}$; also write $P=P_{\mathcal{S}}$. We will now analyse the possible conjugacy class sizes for the noncentral elements of $P$. There are three cases.

(1) First, we consider an element $y \in A_{\alpha_{n}-\alpha_{n-1}}$. Then $C_{P}(y)=\zeta(P) Y=$ $\Phi(X)[X, Y] Y$ and so $\left|y^{P}\right|=|X / \Phi(X)|=p^{\alpha_{1}}$.

(2) Next, we consider an element $y$ in $Y$ but not in $A_{\alpha_{n}-\alpha_{n-1}}$, writing $y=u v$ with $1 \neq u \in P_{\mathcal{S}^{*}}$ and $v \in A_{\alpha_{n}-\alpha_{n-1}}$. Since $v$ is central in $Y$, we have $C_{Y}(y)=$ $C_{P_{\mathcal{S}^{*}}}(u) \times A_{\alpha_{n}-\alpha_{n-1}}$ and $C_{P}(y)=\zeta(P) C_{Y}(y)=\Phi(X)[X, Y] C_{Y}(y)$. It now follows that for some $i \in\{2, \ldots, n-1\}$ we have

$$
\begin{aligned}
\left|y^{P}\right| & =|X / \Phi(X)|\left|Y / C_{Y}(y)\right|=|X / \Phi(X)|\left|P_{\mathcal{S}^{*}} / C_{P_{\mathcal{S}^{*}}}(u)\right| \\
& =|X / \Phi(X)|\left|C l_{P_{\mathcal{S}^{*}}}(u)\right|=p^{\alpha_{1}} p^{\alpha_{i}-\alpha_{1}}=p^{\alpha_{i}} .
\end{aligned}
$$

For $i \in\{2, \ldots, n-1\}$ our induction hypothesis yields an element $w \in P_{\mathcal{S}^{*}}$ with $p^{\alpha_{i}-\alpha_{1}}$ conjugates in $P_{\mathcal{S}^{*}}$. The preceding calculation shows that $\left|w^{P}\right|=p^{\alpha_{i}}$, and therefore the conjugacy class sizes for the noncentral elements in $Y \backslash A_{\alpha_{n}-\alpha_{n-1}}$ are precisely $p^{\alpha_{2}}, p^{\alpha_{3}}, \ldots$, and $p^{\alpha_{n-1}}$.

(3) Finally, we consider an element $u$ of $P=P_{\mathcal{S}}$ not in $Y \zeta(P)$. Then $u=x y z$ with $x \in X \backslash \Phi(X)=X \backslash \zeta(X), y \in Y$ and $z \in[X, Y]$. Suppose $u^{\prime}=x^{\prime} y^{\prime} z^{\prime} \in C_{P}(u)$, with $x^{\prime} \in X, y^{\prime} \in Y$ and $z^{\prime} \in[X, Y]$. Now since $\Phi(P)=\Phi(X) \times \Phi(Y) \times[X, Y]$ and $\left[u, u^{\prime}\right]=\left[x, x^{\prime}\right]\left[y, x^{\prime}\right]\left[x, y^{\prime}\right]\left[y, y^{\prime}\right]=1$ we have $\left[x, x^{\prime}\right]=1$ and $\left[x, y^{\prime}\right]=\left[x^{\prime}, y\right]$. Then since $X$ is free and $x \notin \Phi(X)$ we have $x^{\prime} \in x^{k} \Phi(X)$ for some integer $k$ and then $\left[x, y^{\prime}\right]=\left[x^{k}, y\right]=\left[x, y^{k}\right]$. It now follows that $y^{\prime} y^{-k} \in C_{Y}(x)=\Phi(Y)$. Thus $u^{\prime} \in x^{k} y^{k} \zeta(P)=(x y)^{k} \zeta(P)$ and so $C_{P}(u)=\zeta(P)\langle u\rangle$ and therefore $\left|u^{P}\right|=$ $p^{\alpha_{1}-1+\alpha_{n-1}-\alpha_{1}+1+\alpha_{n}-\alpha_{n-1}}=p^{\alpha_{n}}$.

Thus we have shown that $c s\left(P_{\mathcal{S}}\right)=\mathcal{S}$. This completes the induction step and with it the proof of the theorem.

\section{REFERENCES}

1. I. M. Isaacs, Sets of p-powers as irreducible character degrees, Proceedings of the Amer. Math. Soc. 96 (1986), 551-552. MR 87d:20013

2. Hanna Neumann, Varieties of groups, Springer-Verlag, Berlin, 1967. MR 35:6734

Department of Mathematics, School of Mathematical Sciences, Australian National University, Canberra, 0200, Australia

E-mail address: John.Cossey@maths.anu.edu.au

Mathematics Institute, University of Warwick, Coventry CV4 7AL, United Kingdom

E-mail address: toh@maths.warwick.ac.uk 